point improvement of Lequesne index (difference clinically significant) between groups.

Conclusion Viscosupplementation by Synvisc improves algofunctional status of patients and has no significant impact on specific knee OA cost.

Acknowledgement Synvisc Experts Group: Prs. Bardin T., Dougados M., Kahan A. (Paris), Mazières B (Toulouse), Trèves R. (Limoges), Vignon E. (Lyon), Drs Auvinet B. (Laval), Naim C. (Marseille), Rossignol O. (Fontenay).

\section{SAT0075 EFFICACY OF INTRA-ARTICULAR HYALURONIC ACID INJECTIONS IN KNEE OSTEOARHRITIS}

F Ardic, D Bolulu, O Topuz, S Cubukcu. Physical Medicine and Rehabilitation, Pamukkale University Medical Faculty, Denizli, Turkey

\subsection{6/annrheumdis-2001.450}

\section{Background}

Objectives Our objective was to assess the effects of intra-articular injections of hyaluronic acid (HA) (Hylan G-F 20: Synvisc) on symptoms and functional outcome of knee osteoarthritis (OA).

Methods A total of 60 injections were performed in 17 patients involving 20 knees. These patients with symptomatic, radiological knee OA were randomly assigned to treatment with HA or placebo. Treatment group consisted of 12 patients received three weekly injections of HA into one or both (if bilateral symptomatic) knees (15 knees total). Control group consisted of 5 patients who received three intra-articular injections of 2-ml physiological saline with weekly intervals ( 5 knees total). Fifteenminute walking period, range of motion, need of drug and painless walking time were noted at weeks 1,2,3,8. Pain at walking, resting, night and motion were evaluated by Visual Analogue Scale (VAS). Also, WOMAC Osteoarthritis Indexes (Pain by WOMAC-A, stiffness by WOMAC-B, activities of daily living by WOMAC-C) were evaluated by Likert scales in the same weekly intervals.

Results Statistically significant improvements $(p<0.001)$ in WOMAC-A-3 (3rd week), WOMAC-A-8(8th week), WOMACB-3, WOMAC-B-8, VAS walking-2,3,8, VAS resting? 8, VAS motion-2,3,8, painless walking time-8,15 min walking period-8, need of drug-2,3,8 were found.

When compared with control group, statistically significant improvement was noted ( $\mathrm{p}<0.05$ ) at WOMAC-A-8, WOMACC-8, VAS walking- 8 , VAS resting ?3,8 painless walking time$2,3,8$, need of drug- 8 . No side effect was encountered in both of the groups.

Conclusion Early signs of efficacy of intra-articular injections of HA for knee OA was beginning in the 2nd week. But this early improvement is not significant when compared with control group. The real effects of the HA treatment were seen at the 8th week. It is concluded that Hylan G-F 20 provided good clinical benefit for knee OA patients and the clinical improvement was prominent after 8 weeks.
SAT0076 PATIENTS WITH OSTEOARTHRITIS (OA) REPORT MORE SEVERE PAIN THAN AGE- AND GENDER MATCHED RHEUMATOID ARTHRITIS (RA) PATIENTS

${ }^{1} \mathrm{~B}$ Slatkowsky-Christensen, ${ }^{2} \mathrm{~N}$ Bellamy, ${ }^{1} \mathrm{TK}$ Kvien. ${ }^{1}$ Department of Rheumatology, Diakonhjemmet Hospital, Oslo, Norway; ${ }^{2}$ Centre of National Research on Disability and Rehabilitation Medicine, University of Queensland, Brisbane, Australia

\subsection{6/annrheumdis-2001.451}

Background Patients with RA generally receive more attention by rheumatologists than patients with widespread OA. We wanted to examine if this difference in level of care is based on differences in severity of health status.

Objectives To compare age- and sex matched OA and RA patients regarding levels health status.

Methods Fifty-one patients between 50 and 70 years of age with generalised osteoarthritis (OA) including hand OA, previously referred to a rheumatology outpatient department, underwent a comprehensive clinical examination including completion of several self-reported health status questionnaires. Among these were AIMS2, SF-36, MHAQ, pain and fatigue on VAS. These OA patients were compared with 51 RA patients matched for age and gender. The RA patients were recruited from the Oslo RA register and had previously completed identical questionnaires. Groups were compared with the McNemar tests (categorical variables) and paired analyses (continuous variables).

Results Mean (SD) age was 66.1 (4.4) years, 4(8\%) were males. Pain measures were numerically higher in OA than RA with statically significant difference for 2 (AIMS2 and SF-36) out of 3 measures (Table 1). No statistically significant differences were found within the dimensions of physical and mental health, social functioning and self-efficacy.

\begin{tabular}{lll}
\multicolumn{2}{l}{ Abstract SAT0076 Table 1} & \\
\hline & OA $(\mathbf{n}=51)$ & RA $(\mathbf{n}=51)$ \\
\hline Pain VAS & $40.7(19.3)$ & $37.5(23.2)$ \\
AIMS2 pain & $5.85(2.03)$ & $4.66(2.50)$ \\
SF-36 pain & $37.8(17.0)$ & $46.3(21.5)$ \\
MHAQ & $1.48(0.37)$ & $1.61(0.60)$ \\
SF-36 physical & $54.3(21.3)$ & $45.1(24.4)$ \\
AIMS2 physical & $1.94(1.09)$ & $2.47(1.57)$ \\
SF-36 mental & $68.0(22.7)$ & $70.7(21.9)$ \\
AIMS2 affect & $3.65(1.85)$ & $3.22(1.78)$ \\
\hline Comparison health status (mean (SD) values).
\end{tabular}

Conclusion OA patients report more severe pain than RA patients matched for age and gender, whereas other dimensions of health status appear to be similar, with a tendency of worse physical health in RA and worse mental health in OA patients.

\section{SAT0077 EXAMINATIONS OF THE NORWEGAIN VERSION OF AUSCAN ? A DISEASE SPECIFIC MEASURE OF HAND OSTEOARTHRITIS (OA)}

${ }^{1} \mathrm{~B}$ Slatkowsky-Christensen, ${ }^{2} \mathrm{~N}$ Bellamy, ${ }^{1} \mathrm{TK}$ Kvien. ${ }^{1}$ Department of Rheumatology, Diakonhjemmet Hospital, Oslo, Norway; ${ }^{2}$ Centre of National Research on Disability and Rehabilitation Medicine, University of Queensland, Brisbane, Australia

10.1136/annrheumdis-2001.452

Background In clinical practice we have observed that patients with hand OA frequently have severe pain, stiffness and physical 